\title{
Patient and case characteristics associated with 'no paramedic treatment' for low-acuity cases referred for emergency ambulance dispatch following a secondary telephone triage: a retrospective cohort study
}

Kathryn Eastwood ${ }^{1,2^{*}}$ (D) Amee Morgans ${ }^{3}$, Johannes Stoelwinder ${ }^{1}$ and Karen Smith ${ }^{1,2,4}$

\begin{abstract}
Background: Predicting case types that are unlikely to be treated by paramedics can aid in managing demand for emergency ambulances by identifying cases suitable for alternative management pathways. The aim of this study was to identify the patient characteristics and triage outcomes associated with 'no paramedic treatment' for cases referred for emergency ambulance dispatch following secondary telephone triage.

Methods: A retrospective cohort analysis was conducted of cases referred for emergency ambulance dispatch following secondary telephone triage between September 2009 and June 2012. Multivariable logistic regression modelling was used to identify explanatory variables associated with 'no paramedic treatment'.
\end{abstract}

Results: There were 19,041 cases eligible for inclusion in this study over almost three years, of which 8510 (44.7\%) were not treated after being sent an emergency ambulance following secondary triage. Age, time of day, pain, triage guideline group, and comorbidities were associated with 'no paramedic treatment'. In particular, cases $0-4$ years of age or those with psychiatric conditions were significantly less likely to be treated by paramedics, and increasing pain resulted in higher rates of paramedic treatment.

Conclusions: This study highlights that case characteristics can be used to identify particular case types that may benefit from care pathways other than emergency ambulance dispatch. This process is also useful to identify gaps in the alternative care pathways currently available. These findings offer the opportunity to optimise secondary telephone triage services to support their strategic purpose of minimising unnecessary emergency ambulance demand and to match the right case with the right care pathway.

Keywords: Emergency medical services, Triage., Telephone., Emergency medical service communication systems., Ambulance., Health resources.

\footnotetext{
* Correspondence: Kathryn.Eastwood@monash.edu

'Department of Epidemiology and Preventive Medicine, The Alfred Centre,

Monash University, 99 Commercial Road, Melbourne, VIC 3004, Australia

${ }^{2}$ Ambulance Victoria, Victoria, Australia

Full list of author information is available at the end of the article
} 


\section{Background}

The increasing demand for emergency ambulances, particularly from patients not requiring specific paramedic interventions, has been reported in numerous studies [1-9]. This demand places significant strain upon costly and finite emergency ambulance resources $[2,8,10,11]$, and risks the lives and health of patients who need these skills by contributing to resource scarcity [2, 12-14]. In order to manage increasing demand some ambulance services have implemented secondary telephone triage services, whereby specific low-acuity cases undergo a nurse, doctor or paramedic-led telephone triage prior to or concurrent with an emergency ambulance dispatch $[13,15-22]$. The goal of this triage is to reduce the demand for emergency ambulances by diverting suitable cases to alternative transportation or management pathways $[15,23]$. The impact upon ambulance demand has varied, with some services referring up to $71 \%$ of the cases triaged for emergency ambulance dispatch [17]. The Referral Service, a secondary telephone triage service operated by Ambulance Victoria in Victoria, Australia, referred $27.6 \%$ of its cases for emergency ambulance dispatch between 2009 and 2012 [15, 24]. Whilst these decisions may have been appropriate, $44.7 \%$ of these cases were not treated by paramedics, suggesting there is opportunity for optimisation of triage processes. Using emergency ambulance resources for cases not requiring specific paramedic treatment when other options exist is an inappropriate and costly use of resources and does not provide the most suitable care for these patients.

Primary triage tools such as the Medical Priority Dispatch System have been investigated to identify cases not likely to be treated by advanced life support (ALS) paramedics, however most have used simple measures such as frequencies $[4,5,7,9]$, likelihood ratios and predictive values $[3,6,8]$, which do not allow for associations or relationships to be ascertained accurately. Furthermore, investigating primary triage tools for case types associated with 'no paramedic treatment' is impractical because these triage tools are designed to rapidly dispatch ambulances and therefore lack granularity in their assessment of cases. Unsurprisingly, the few studies examining triage of low-acuity patients have achieved only moderate success in identifying cases unlikely to be treated by ALS paramedics [2-8]. In summary, there is little reliable research investigating the associations between paramedic treatment, low-acuity patient characteristics and triage outcomes.

Ambulance-based secondary triage systems that utilise less time-sensitive, more granular triage tools which are applied by healthcare clinicians presents a better opportunity to investigate low-acuity cases and their association with ALS paramedic treatment. Identifying cases unlikely to be treated by paramedics that appear suitable for diversion to alternative forms of transportation or care can potentially reduce the demand for emergency ambulances and better align appropriate care pathways with patient need. The aim of this study was to identify the patient characteristics and triage outcomes associated with 'no paramedic treatment' for cases referred for emergency ambulance dispatch following secondary telephone triage.

\section{Methods \\ Study design}

A retrospective cohort analysis was conducted of cases referred for emergency ambulance dispatch following secondary telephone triage between September 2009 and June 2012.

\section{Study setting}

Ambulance Victoria is the sole emergency medical service (EMS) provider in the state of Victoria, Australia. Melbourne is the capital and largest city in Victoria, and during the study timeframe Ambulance Victoria responded to 1,036,114 cases in Melbourne, which had a population of 4.25 million in 2012 [25, 26]. Ambulance Victoria uses a two-tiered medical response system to respond to calls for emergency medical assistance. ALS paramedics form the base qualification level, and mobile intensive care ambulance (MICA) paramedics comprise the upper tier, possessing further interventional skills and a broader suite of pharmacological agents to treat patients [27]. The decision to respond ALS paramedics, MICA paramedics or both is made using the Advanced Medical Priority Dispatch System (AMPDS) primary triage tool and an Ambulance Victoria formulated service allocation matrix [15].

The Referral Service, a subsidiary of Ambulance Victoria, has operated $24 \mathrm{~h}$ a day, 7 days a week since 2003, providing secondary telephone triage to cases identified as low-acuity following primary triage. These low-acuity cases are specific case types that Ambulance Victoria has identified previously as having low paramedic treatment and transportation rates and that rarely re-present with the ambulance service within $24 \mathrm{~h}$ [28-30] (Ambulance Victoria: Referral Service Review: The case for expansion, unpublished report). Cases that are identified as low-acuity following the AMPDS-led primary triage are transferred to the Referral Service instead of an immediate emergency ambulance dispatch. Cases undergo secondary telephone triage by qualified paramedics or nurses using a computer-based triage algorithm to determine the most appropriate disposition for the case. This may involve the provision of self-care advice, advice to self-present at a community-based 
medical or health service or hospital, the dispatch of an alternative service provider including home-visiting doctors, nurses and hospital outreach programs, or the dispatch of a non-emergency ambulance or emergency ambulance. During the study period the Referral Service triaged 103,768 low-acuity cases in metropolitan Melbourne. The Referral Service have been described in more detail elsewhere [15].

\section{Definitions}

Local protocols have been utilised in other studies to define ALS and basic life support (BLS) levels of paramedic treatment, and the latter has also been used to define low-acuity cases [4, 6-8]. In Victoria, non-emergency ambulance officers are able to provide BLS care, including cardiac monitoring, extrication and manual handling assistance, basic first aid and utilise a small range of drugs to manage patients. Therefore in Victoria, lowacuity cases that still require transportation to an emergency department should be suitable for referral to a non-emergency ambulance following secondary telephone triage.

In this study, 'no paramedic treatment' was defined as being when no prehospital treatment or only BLS treatment occurred (i.e. no ALS paramedic level of treatment was required). Intravenous line insertion, when it was not subsequently used for drug or fluid resuscitation, was excluded from the ALS level of treatment, which aligns with previous studies $[3,4,6]$. Paramedic treatment therefore consisted of drug or fluid administration, airway management (including oxygen therapy through to airway adjuncts), perfusion or cardiovascular support management, and mental health management including chemical or physical restraint.

\section{Data sources}

Referral Service records were extracted from the Referral Service database and corresponding paramedic records for the cases referred for emergency ambulance dispatch were extracted and linked. These records documented patient assessment, treatment, demographic and operational information.

\section{Procedures}

Only cases with a secondary telephone triage record and a corresponding paramedic care record were included in the study. The explanatory variables selected were patient age, gender, income status, comorbidities, pain score, triage guideline, time of call, and call-taker qualification. These were selected based on their availability in the datasets and likely association with the outcome. The categories investigated within each of these variables are listed in Table 1. The 343 triage guidelines used to triage the cases directed to the Referral Service were
Table 1 Explanatory variables used in the prediction modelling

\begin{tabular}{|c|c|}
\hline Variable name & Reference category \\
\hline Age & $\begin{array}{l}\text { 55-59 year olds (selected because it contained the } \\
\text { mean age for the cases included in this study) }\end{array}$ \\
\hline Gender & Males \\
\hline Income status & The 'above' category \\
\hline Qualification & $\begin{array}{l}\text { ALS paramedics (because they triaged the largest } \\
\text { volume of cases) }\end{array}$ \\
\hline Pain level & Mild \\
\hline Time of day & Day shift \\
\hline $\begin{array}{l}\text { Triage guideline } \\
\text { groups }\end{array}$ & $\begin{array}{l}\text { Given the outcome variable involved the presence } \\
\text { or absence of ALS paramedic treatment, the } \\
\text { category with the most similar paramedic treatment } \\
\text { rate to all Victorian emergency ambulance cases } \\
\text { (55.5\%) was chosen to compare with the triage } \\
\text { guideline groups - the urinary symptoms group } \\
\text { (paramedic treatment rate of } 55.2 \% \text { ) }\end{array}$ \\
\hline
\end{tabular}

consolidated into 44 triage guideline groups to improve model parameter approximations and maintain the predictive power of the logistic regression [31, 32]. The 21 guideline groups with the highest frequency of usage were utilised for this study to aid identification of relationships with the greatest impact. These guideline groups contained $80.4 \%$ of the cases eligible for inclusion in this study. Using these guideline groups ensured sufficient group populations for reliable results.

The median wage in local government areas (LGAs) was converted to a binary variable, consisting of LGAs with a median wage above or below that of the median metropolitan Melbourne wage (AU\$54,100) in 2011-12. Similarly, the time of day variable was dichotomised to correspond to the day shift (0700-1700 h) and night shift (1700-0700 h) roster that most emergency ambulance crews worked at the time of the study. The call-taker qualification variable distinguished qualified nurses, ALS paramedics and MICA paramedics. Finally pain scores recorded on a scale of 1-10 were divided into three groups: mild pain $<3$, moderate pain 3-6 and severe pain 7-10. According to the local protocols, any patient in the moderate or severe pain group was expected to have been treated with analgesia unless the patient declined treatment.

The reference categories for the seven categorical variables are listed and explained in Table 1.

A systematic bias evaluation was conducted, involving comparison of age, gender and triage guideline groups for cases that had a corresponding paramedic record and those that did not (but where the triage outcome indicated an ambulance had been dispatched). The gender distribution did not differ between these groups (54.9\% versus $55.1 \%$ respectively; chi-square $=0.093, \mathrm{df}=1, p=0.760$ ). However, these groups had different mean ages (57.6 years versus 49.8 years; $\mathrm{t}(8885.5) 21.85, p<0.001)$ and 
triage guideline groups (chi-square $=411.567, \mathrm{df}=44$, $p<0.001$ ). The most common triage guideline groups for the cases with a corresponding paramedic record were abdominal pain (18.2\%), back pain $(9.9 \%)$ and dizziness and vertigo (6.7\%). For the cases referred to an emergency ambulance without a corresponding paramedic record, the most common triage guideline groups were abdominal pain (24.2\%), back pain (9.1\%) and psychiatric conditions (7.8\%). It should be noted that a large sample size can result in high statistical sensitivity to small distribution differences, which may be apparent in the statistical difference in the triage guideline groups. Nonetheless, this systematic bias evaluation does suggest that the missing cases may have imposed some bias.

\section{Statistical analysis}

This study utilised descriptive statistical analysis and multivariable binary logistic regression analyses to determine whether there were associations between the explanatory variables and the paramedic treatment outcome variable. All the explanatory variables were assessed for multiple collinearity and found to have no correlation likely to affect the multivariable analysis [33]. All statistical analysis was conducted using SPSS Version 23 [34].

\section{Results}

Ambulance Victoria received over one million calls to triple zero during the study timeframe and 103,768 cases had a complete Referral Service triage during this time. Of these cases, 29,579 (28.5\%) were referred for an emergency ambulance dispatch. Figure 1 outlines the case selection process that resulted in 19,041 cases being eligible for inclusion.

There were 8510 cases $(44.7 \%)$ were not treated by attending paramedics after having an emergency ambulance dispatched following secondary telephone triage. This 'no paramedic treatment' cohort of cases averaged 59.3 years of age (SD 25.3 years) and $54.4 \%$ were female.

The multivariable logistic regression model shown in Table 2 identifies that patient age, pain, triage guideline group, time of day and comorbidities variables were associated with 'no paramedic treatment' $(p<0.001)$. The reference categories for these variables were 55-59 year old patients (mean age), pain $<3 / 10$, urinary symptoms (which had a paramedic treatment rate most similar to all emergency ambulance cases), day shift and no comorbidities respectively. Compared to these reference categories, some of the other categories within these variables demonstrated a significant relationship with 'no paramedic treatment'. Call-taker qualification $(p=0.816)$ and income status $(p=0.544)$ demonstrated no association with 'no paramedic treatment'.

Patients under the age of 25 years were significantly more likely to receive 'no paramedic treatment' than 55-59 year olds (Table 2). The 'no paramedic treatment' rates in cases under 15 years of age ranged from 52.0$92.7 \%$ within 5 -year age groups (Table 2). Cases occurring overnight were more likely to go untreated by paramedics (OR 1.17, 95\% CI 1.08-1.26; $p<0.001$ ) than cases that occurred during the day. With each additional comorbidity, patients became less likely to be untreated

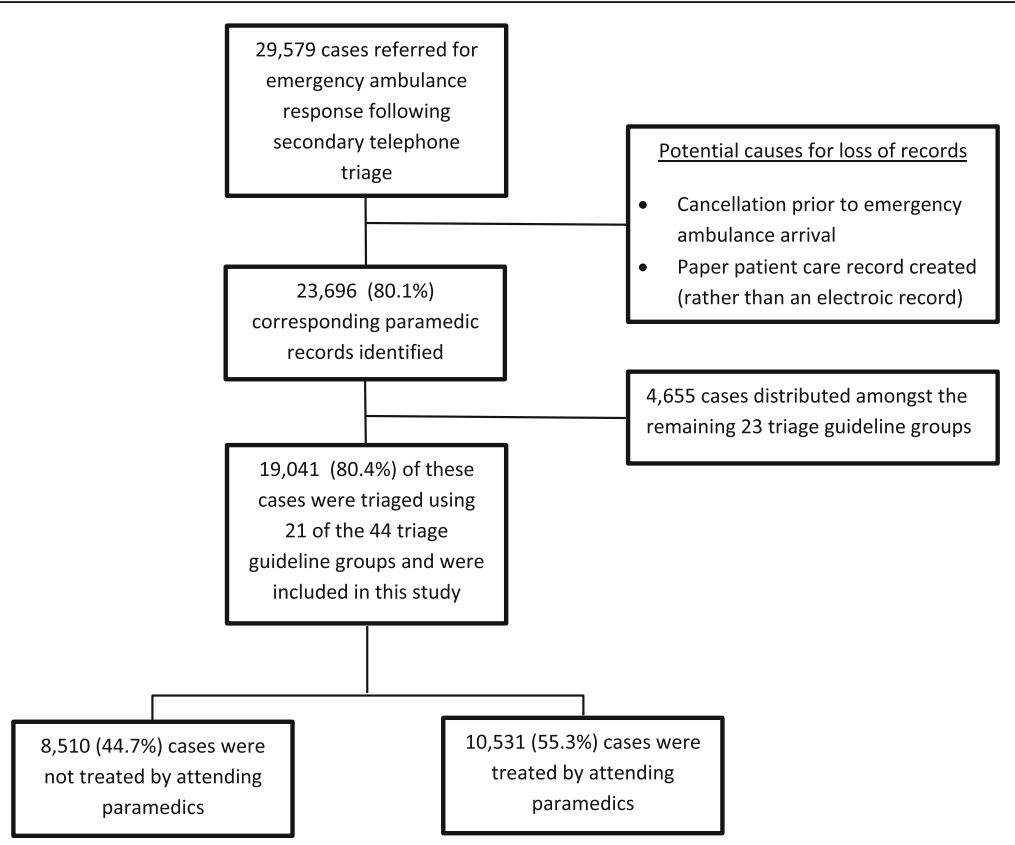

Fig. 1 Case-flow inclusion chart 
Table 2 Univariable and multivariable analyses of the explanatory variables being assessed for their association with 'no paramedic treatment'

\begin{tabular}{|c|c|c|c|c|c|c|}
\hline \multirow[b]{2}{*}{ Explanatory variable } & \multirow[b]{2}{*}{ Categories } & \multirow[b]{2}{*}{ Frequency } & \multirow[b]{2}{*}{$\begin{array}{l}\% \text { not treated by } \\
\text { paramedics }(95 \% \mathrm{Cl})\end{array}$} & \multicolumn{3}{|c|}{ Multivariable logistic regression } \\
\hline & & & & OR $(95 \% \mathrm{Cl})$ & Category $p$-value & Variable $p$-value \\
\hline \multirow[t]{2}{*}{ Gender } & Male & 7700 & $45.2(44.2-46.3)$ & 1 & - & 0.477 \\
\hline & Female & 9256 & $44.2(43.3-45.2)$ & $1.03(0.95-1.11)$ & 0.477 & \\
\hline \multirow[t]{18}{*}{ Age } & $0-4$ yrs & 300 & $92.7(89.7-95.6)$ & $6.14(3.81-9.88)$ & $<0.001$ & $<0.001$ \\
\hline & $5-9$ yrs & 182 & $69.2(62.5-76.0)$ & $2.46(1.62-3.74)$ & $<0.001$ & \\
\hline & $10-14$ yrs & 202 & $52.0(45.0-58.9)$ & $1.51(1.01-2.26)$ & 0.047 & \\
\hline & $15-19$ yrs & 436 & $46.3(41.6-51.0)$ & $1.61(1.18-2.20)$ & 0.003 & \\
\hline & $20-24$ yrs & 656 & $45.6(41.8-49.4)$ & $1.40(1.07-1.83)$ & 0.015 & \\
\hline & $25-29$ yrs & 847 & $39.3(36.0-42.6)$ & $1.24(0.96-1.60)$ & 0.098 & \\
\hline & $30-34$ yrs & 1006 & $38.8(35.8-41.8)$ & $1.27(0.99-1.61)$ & 0.057 & \\
\hline & $35-39$ yrs & 1041 & $34.6(31.7-37.5)$ & $1.10(0.87-1.40)$ & 0.425 & \\
\hline & $40-44$ yrs & 1195 & $35.5(32.8-38.2)$ & $1.19(0.94-1.50)$ & 0.150 & \\
\hline & $45-49$ yrs & 959 & $35.1(32.1-38.2)$ & $1.01(0.79-1.29)$ & 0.927 & \\
\hline & $50-54$ yrs & 932 & $38.3(35.2-41.4)$ & $0.94(0.74-1.18)$ & 0.580 & \\
\hline & $55-59$ yrs & 981 & $39.8(36.7-42.8)$ & 1 & - & \\
\hline & $60-64$ yrs & 1183 & $40.2(37.4-43.0)$ & $0.97(0.78-1.21)$ & 0.793 & \\
\hline & $65-69$ yrs & 1280 & $45.3(42.6-48.0)$ & $1.06(0.86-1.31)$ & 0.589 & \\
\hline & 70-74 yrs & 1519 & $43.8(41.4-46.3)$ & $0.97(0.79-1.19)$ & 0.758 & \\
\hline & 75-79 yrs & 1782 & $47.1(44.8-49.4)$ & $1.04(0.85-1.26)$ & 0.732 & \\
\hline & $80-84$ yrs & 2056 & $50.5(48.3-52.7)$ & $1.18(0.97-1.42)$ & 0.101 & \\
\hline & $85+y r s$ & 2484 & $52.8(50.8-54.7)$ & $1.16(0.96-1.40)$ & 0.124 & \\
\hline \multirow[t]{2}{*}{ Time of day } & 0700-1859 h & 11,621 & $44.6(43.7-45.5)$ & 1 & - & $<0.001$ \\
\hline & 1900-0659 h & 7420 & $44.8(43.7-46.0)$ & $1.17(1.08-1.26)$ & $<0.001$ & \\
\hline \multirow[t]{2}{*}{$\begin{array}{l}\text { Income status of } \\
\text { patients LGA }\end{array}$} & $\begin{array}{l}\text { Above median } \\
\text { income of } \$ 54 \mathrm{~K}\end{array}$ & 2499 & $47.6(45.7-49.6)$ & 1 & - & 0.544 \\
\hline & $\begin{array}{l}\text { Below median } \\
\text { income of } \$ 54 \mathrm{~K}\end{array}$ & 16,542 & $44.3(43.5-45.0)$ & $1.03(0.93-1.15)$ & 0.544 & \\
\hline \multirow[t]{3}{*}{ Pain level } & Mild (pain <3/10) & 11,139 & $68.6(67.7-69.4)$ & 1 & - & $<0.001$ \\
\hline & $\begin{array}{l}\text { Moderate (pain } \\
3-6 / 10)\end{array}$ & 2696 & $24.0(22.4-25.6)$ & $0.16(0.14-0.18)$ & $<0.001$ & \\
\hline & Severe (pain >6/10) & 5206 & $4.4(3.8-4.9)$ & $0.02(0.02-0.03)$ & $<0.001$ & \\
\hline \multirow{12}{*}{$\begin{array}{l}\text { Triage guideline } \\
\text { groups }\end{array}$} & Abdominal pain & 4276 & $27.2(25.9-28.5)$ & $0.83(0.63-1.08)$ & 0.158 & $<0.001$ \\
\hline & Back pain & 2319 & $22.3(20.5-23.9)$ & $0.79(0.60-1.04)$ & 0.098 & \\
\hline & Dizziness & 1566 & $58.4(56.2-61.1)$ & $0.77(0.58-1.01)$ & 0.056 & \\
\hline & Nausea / vomiting & 1320 & $54.0(51.4-56.7)$ & $0.72(0.55-0.95)$ & 0.022 & \\
\hline & Psychiatric conditions & 1087 & $89.8(87.9-91.5)$ & $4.44(3.19-6.18)$ & $<0.001$ & \\
\hline & Fever & 1068 & $62.9(60.1-65.8)$ & $0.86(0.64-1.14)$ & 0.287 & \\
\hline & Weakness & 907 & $64.7(61.6-67.8)$ & $1.05(0.79-1.40)$ & 0.749 & \\
\hline & Headache & 839 & $42.0(38.6-45.3)$ & $1.18(0.87-1.61)$ & 0.287 & \\
\hline & $\begin{array}{l}\text { Confusion/Disorientation } \\
\text { /Agitation }\end{array}$ & 753 & $65.7(62.3-69.0)$ & $1.01(0.75-1.35)$ & 0.966 & \\
\hline & Flank Pain & 663 & $14.9(12.3-17.8)$ & $0.68(0.47-0.97)$ & 0.035 & \\
\hline & Hip Non-Injury & 526 & $22.2(18.9-26.1)$ & $0.65(0.46-0.92)$ & 0.016 & \\
\hline & Chest Pain / Discomfort & 497 & $38.5(34.3-42.8)$ & $0.58(0.42-0.81)$ & 0.001 & \\
\hline
\end{tabular}


Table 2 Univariable and multivariable analyses of the explanatory variables being assessed for their association with 'no paramedic treatment' (Continued)

\begin{tabular}{|c|c|c|c|c|c|c|}
\hline \multirow{2}{*}{ Explanatory variable } & \multirow[b]{2}{*}{ Categories } & \multirow[b]{2}{*}{ Frequency } & \multirow[b]{2}{*}{$\begin{array}{l}\% \text { not treated by } \\
\text { paramedics }(95 \% \mathrm{Cl})\end{array}$} & \multicolumn{3}{|c|}{ Multivariable logistic regression } \\
\hline & & & & OR $(95 \% \mathrm{Cl})$ & Category $p$-value & Variable $p$-value \\
\hline & $\begin{array}{l}\text { Neurological Symptoms/ } \\
\text { Transient Ischaemic Attack }\end{array}$ & 436 & $58.3(53.7-62.9)$ & $0.76(0.55-1.05)$ & 0.097 & \\
\hline & Trauma & 415 & $47.0(42.2-51.8)$ & $1.21(0.85-1.72)$ & 0.301 & \\
\hline & Fainting & 370 & $61.8(56.9-66.7)$ & $0.79(0.56-1.12)$ & 0.179 & \\
\hline & Hypertension & 370 & $69.4(64.7-74.1)$ & $1.46(1.03-2.07)$ & 0.033 & \\
\hline & Hip Injury & 324 & $27.8(23.0-32.7)$ & $0.60(0.41-0.88)$ & 0.009 & \\
\hline & $\begin{array}{l}\text { Diabetes-related } \\
\text { problems }\end{array}$ & 319 & $52.6(47.2-58.1)$ & $0.78(0.55-1.11)$ & 0.169 & \\
\hline & $\begin{array}{l}\text { Musculoskeletal } \\
\text { other }\end{array}$ & 311 & $50.8(45.2-56.4)$ & $1.34(0.91-1.95)$ & 0.136 & \\
\hline & Diarrhoea & 310 & $64.9(59.5-70.2)$ & $1.20(0.84-1.73)$ & 0.316 & \\
\hline & Urinary symptoms & 365 & $44.8(39.7-49.9)$ & 1 & - & \\
\hline \multirow{3}{*}{$\begin{array}{l}\text { Referral Service } \\
\text { call-taker qualification }\end{array}$} & Nurse & 2700 & $45.3(43.4-47.1)$ & $1.01(0.91-1.12)$ & 0.903 & 0.816 \\
\hline & ALS paramedic & 15,813 & $44.7(43.9-45.5)$ & 1 & - & \\
\hline & $\begin{array}{l}\text { Intensive care } \\
\text { paramedic }\end{array}$ & 528 & $41.1(36.9-45.3)$ & $0.93(0.74-1.17)$ & 0.538 & \\
\hline $\begin{array}{l}\text { Continuous } \\
\text { variables }\end{array}$ & $\begin{array}{l}\text { Mean number of } \\
\text { comorbidities for } \\
\text { those treated (SD) }\end{array}$ & & $\begin{array}{l}\text { Mean number of } \\
\text { comorbidities for } \\
\text { those not treated (SD) }\end{array}$ & & & \\
\hline Comorbidities & 3.15 (SD 2.3) & & $2.96(2.2)$ & $0.92(0.91-0.94)$ & $<0.001$ & $<0.001$ \\
\hline
\end{tabular}

(OR 0.92, 95\% CI 0.91-0.94; $p<0.001$ ). Seven of the triage guideline groups demonstrated a significant association with 'no paramedic treatment' when compared to the reference group (Table 2). Finally, increasing pain was associated with decreasing rates of 'no paramedic treatment' (Table 2). Overall the multivariable analysis explained $47.3 \%$ of the variation in the outcome and the ability of the model to correctly predict the outcome was $78.6 \%$. The sensitivity of the model was good at $87.3 \%$ and the specificity was $72.3 \%$.

Of those categories demonstrating a statistically significant association with 'no paramedic treatment', the paediatric cases, psychiatric condition cases and pain cases showed the greatest association with this outcome and greatest potential for impact upon demand. The strongest association occurred for the 0-4 year old category, who were over six times more likely to be left untreated (OR 6.14, 95\% CI 3.81-9.88; $p<0.001$ ) than the 55-59 year old category. The mean age for cases aged $0-4$ years was 1.6 years; only $7.3 \%$ of cases were treated by paramedics, and they received oxygen therapy (48.3\%; $n=14)$, analgesia $(24.1 \% ; n=7)$ and other drug administration $(10.3 \% ; n=3)$. The most common triage guideline groups used for the 0-4 year old category were fever $(38.8 \%, n=118)$ and nausea and vomiting $(24.7 \%, n=75)$. When the $0-4$ year old cases with fever were compared to the remaining 0-4 year old cases, they demonstrated paramedic treatment rates almost the same as the remaining cases $(8.5 \%$ versus 9.8\%; $\left.\chi^{2}(1)=0.158 ; p=0.691\right)$. However the $0-4$ year old cases with nausea and vomiting had significantly lower rates of paramedic treatment than the remaining $0-4$ year old cases $\left(1.3 \%\right.$ versus $11.9 \%$; $\chi^{2}$ (1) $=7.519 ; p=0.006)$.

Psychiatric condition cases were over four times more likely to be untreated than the reference group (OR 4.44, 95\% CI 3.19-6.18; $p<0.001)$. The mean age for this category was 44.1 years. Further investigation of the age groups in this category demonstrated no association between age and 'no paramedic treatment' $(p=0.854)$. The most common paramedic treatments for the $10.2 \%$ of cases treated in this category were oxygen therapy (49.5\%, $n=55)$, analgesia $(29.7 \%, n=33)$ and mental health management $(15.3 \%, n=17)$. Local protocols have changed since the study period, whereby oxygen therapy is only indicated in patients with an oxygen saturation of $<94 \%$. Had this protocol been active during the study, only two psychiatric condition cases would have been indicated for oxygen therapy.

The severe pain group were $98 \%$ more likely to be treated than patients with mild pain (OR 0.02, 95\% CI $0.02-0.03 ; p<0.001)$, and the moderate pain group were $84 \%$ more likely to be treated than patients with mild pain (OR 0.16, 95\% CI 0.14-0.18; $p<0.001$ ). Mean ages 
for these categories were mild pain: 64.2 years, moderate pain: 56.9 years and severe pain: 51.3 years. The most common paramedic treatments for moderate and severe pain cases were analgesia $(89.1 \%, n=1864$ and $98.2 \%, n=4962$ respectively) and IV access (31.3\%, $n=654$; and $47.6 \%$, $n=2406$ respectively). The mild pain category was most commonly treated with oxygen therapy (58.4\%; $n=1781)$ and IV access (36.8\%; $n=1122)$. Again, had more recent oxygen administration protocols been in place, $47.1 \%$ of the cases that received oxygen in this variable would no longer be indicated for it.

The most common triage guideline groups for patients complaining of mild pain were dizziness (13.1\%; $n=1454)$, abdominal pain $(11.3 \% ; n=1261)$ and nausea and vomiting $(9.6 \% ; n=1070)$. The two most common triage guidelines for moderate and severe pain were abdominal pain (moderate: $33.0 \% ; n=890$; severe: $40.8 \% ; n=2125$ ) and back pain (moderate: $20.3 \% ; n=546$; severe: $23.7 \% ; n=1236$ ). Headache was the third most common triage guideline for moderate pain $(5.7 \% ; n=155)$ and flank pain $(8.4 \% ; n=435)$ was the third triage guideline for severe pain.

\section{Discussion}

This is the first study to use secondary telephone triage records and paramedic records to identify factors associated with 'no paramedic treatment' in patients with an emergency ambulance dispatch. Five of the eight variables investigated were significantly associated with this outcome. Both the 0-4 years' age category and psychiatric conditions triage guideline category had high rates of 'no paramedic treatment', whilst the pain variable demonstrated low rates of 'no paramedic treatment'. Hence these categories appear to offer the most potential for increasing the specificity of triage decisions regarding the likely need for paramedic intervention.

Paediatric cases, particularly those under 5 years old, were over six times less likely to be treated than 55-59 year olds. This is supported by previous work showing paediatric cases had lower rates of paramedic treatment and higher rates of inappropriate usage [35-37]. The triage guideline group used to triage this age group also influenced the paramedic treatment rates, with the nausea and vomiting cases having extremely low paramedic treatment rates (1.3\%), suggesting that emergency ambulance is not the most appropriate option for these cases. An investigation of whether these cases were appropriate for the ED and their temporal trends in ambulance service usage may reveal that access to alternative service providers specifically relating to childhood illness, such as specialist telephone advice lines, may allow for these cases to be managed with other care pathways [37]. Should these cases be found to be suitable for the ED, other forms of transportation to hospital such as nonemergency ambulances or transportation by the child's family (where appropriate) should be considered.

The high rates of 'no paramedic treatment' for psychiatric condition cases are consistent with previous research which found these cases make up a large proportion of low-acuity work for ambulance services [12], and may be more suitable for other forms of transportation to hospital [38]. During the study period Ambulance Victoria was responsible for the transportation of psychiatric cases considered too unwell to be transported by mental health staff alone [39]. The protocol for the transportation of psychiatric cases was adjusted in 2014, after this study's timeframe, to reflect the increasing skill level of non-emergency patient transport attendants, emergency ambulance paramedics and the increasing workload. The revised protocol now states that an emergency ambulance is to be used when the patient's medical needs can only be met by an emergency ambulance service, and includes non-emergency ambulances as a potential option for these cases [40]. The paramedic treatment rate identified in this study supports this shift in responsibility.

Increasing pain was the most significant predictor of 'no paramedic treatment' (Table 2). A UK study of a secondary telephone triage services found a large proportion of pain cases were referred for emergency ambulance dispatch following triage [20]. Pain severity is not explored during the primary triage process and not considered when classifying cases as low-acuity, so the subsequent referral of severe pain cases back to emergency ambulance dispatch was not surprising. Furthermore, the strong relationship between pain and 'no paramedic treatment' was expected given the range of analgesic agents carried by ALS paramedics. This lack of consideration of pain severity has been a source of dissatisfaction with secondary telephone triage for patients, who feel that this process delayed access to analgesia [20]. The assessment of pain during the secondary telephone triage process could be refined to determine whether an expedient alternative response for managing pain could be introduced into the alternative care pathways. This could include a referral to a home-visiting doctor or the development of a pain pathway that allows patients with chronic pain to have a telephone consultation with a pain specialist. This may allow the provision of advice on how to use their current resources and medications to manage this acute episode whilst arranging an appointment with their regular pain management healthcare worker. For patients requiring ED assessment in Victoria, non-emergency ambulance officers have analgesia that could be used during transportation of these patients.

On initial inspection the categories and variables that had high rates of paramedic treatment suggest that these 
cases should have had an emergency ambulance dispatched without secondary telephone triage. However, in some instances these cases may only represent a small proportion of the total number of these case types that underwent a secondary telephone triage, and other cases within this case type may have successfully been referred away from emergency ambulance dispatch. They may constitute a sub-cohort that was successfully identified by the call-takers as requiring emergency ambulance dispatch. Therefore, further investigation is required before any recommendations can be made. Moreover, a study of the care delivered in the ED, and in the primary care setting should be conducted to direct the development of alternative care pathways and identification of cases suitable for these pathways.

Finally, the identification of 'no paramedic treatment' was used in this study, and in others [2-8], to suggest that cases falling into this category are not suitable for emergency ambulance attendance. However, there may be some cases that have low rates of paramedic treatment that are suitable for an emergency ambulance. There are a range of non-clinical indicators for appropriateness when considering emergency ambulance transport [41], which predominantly relate to patient welfare, safety and a responsibility of the ambulance services to ultimately 'do the right thing' as part of a bigger healthcare system [41]. When optimising secondary telephone triage systems, key questions should be incorporated to identify some of these situations (e.g. an unattended minor), and the ability to override the system disposition will ensure that call-takers are able to dispatch an emergency ambulance in situations where they feel it is appropriate.

\section{Limitations}

This was a retrospective analysis using predefined variables that were not primarily devised to ascertain relationships. A potential for systematic bias was exerted by the $19.1 \%$ of cases that could not be linked to a paramedic record. The direction of this bias could not be definitively ascertained, however the lack of a paramedic record may indicate the patient was not transported by paramedics. In developing the logistic regression model, reference categories had to be chosen for comparison between the categories (the rationale for the reference categories is given in the study methodology). This makes the outcomes relative to this category and can make generalisability difficult.

Secondary telephone triage systems embedded in ambulance services differ in their operational structures, alternative service providers and staff qualifications, therefore potentially altering triage outcomes. The operation of ambulance services, and structure of primary healthcare systems within which these ambulance services operate differ between regions influencing the case-mix for secondary telephone triage. These factors may limit the generalisability of the results.

Finally, the researchers acknowledge that using paramedic treatment to identify cases suitable for alternative management streams does not take into consideration the non-clinical management skills and knowledge paramedics have, and alternative reasons why a patient might be considered suitable for an emergency ambulance dispatch. Similarly, the definition of low-acuity used in this study was limited to emergency ambulance suitability, and no ED or hospital outcomes were included in the definition.

\section{Conclusion}

This study has highlighted that case characteristics can be used to identify particular case types that may benefit from care pathways other than emergency ambulance dispatch. This process is also useful to identify gaps in the alternative care pathways that may allow some case types to avoid entering the emergency care pathways. These findings offer the opportunity to optimise secondary telephone triage services to achieve their strategic purpose of minimising unnecessary emergency ambulance demand and match the right case with the right care pathway.

\section{Abbreviations \\ ALS: Advanced life support; AMPDS: Advanced Medical Priority Dispatch System; BLS: Basic life support; EMS: Emergency medical service; IV: Intravenous; LGA: Local government area; MICA: Mobile intensive care ambulance}

\section{Acknowledgements}

Sam Brilleman and Professor Rory Wolfe for their assistance in the statistical analysis.

\section{Funding}

Not applicable.

\section{Availability of data and materials}

Data sharing: no additional data available.

\section{Authors' contributions}

KE: study conception, data analysis, wrote the paper. AM: discussed core ideas, co-wrote the paper. JS: Discussed core ideas, edited the paper. KS: discussed core ideas, assisted in the data analysis, co-wrote the paper. KS: is Ms. Eastwood's primary PhD supervisor. All authors read and approved the final manuscript.

\section{Ethics approval and consent to participate}

Ethical approval was granted by the Monash University Human Research Ethics Committee (CF12/0547-2,012,000,215), and organisational approval by the Ambulance Victoria Research Governance Committee (R11-021).

No patients were directly accessed, therefore no consent was required.

\section{Consent for publication}

Not applicable.

\section{Competing interests}

Kathryn Eastwood is an intensive care paramedic who intermittently works for the Ambulance Victoria Referral Service (secondary telephone triage service). Professor Johannes Stoelwinder has previously been the Chair of the 
Board of Ambulance Victoria. Professor Karen Smith is the Director of the Centre for Research and Evaluation at Ambulance Victoria.

\section{Publisher's Note}

Springer Nature remains neutral with regard to jurisdictional claims in published maps and institutional affiliations.

\section{Author details}

'Department of Epidemiology and Preventive Medicine, The Alfred Centre, Monash University, 99 Commercial Road, Melbourne, VIC 3004, Australia ${ }^{2}$ Ambulance Victoria, Victoria, Australia. ${ }^{3}$ Emergency Services Telecommunications Authority, Victoria, Australia. ${ }^{4}$ Department of Community Emergency Health and Paramedic Practice, Monash University, Melbourne, VIC, Australia.

\section{Received: 21 October 2017 Accepted: 3 January 2018}

\section{Published online: 10 January 2018}

\section{References}

1. Uscher-Pines L, Pines J, Kellermann A, Gillen E, Mehrotra A. Deciding to visit the emergency Department for non-Urgent Conditions: a systematic review of the literature. Am J Manag Care. 2013;19(1):47-59.

2. Grusd E, Kramer-Johansen J. Does the Norwegian emergency medical dispatch classification as non-urgent predict no need for pre-hospital medical treatment? An observational study. Scand J Trauma Resusc Emerg Med. 2016;24(1):65

3. Sporer KA, Craig AM, Johnson NJ, Yeh CC. Does emergency medical dispatch priority predict delphi process-derived levels of prehospital intervention? Prehospital Disaster Med. 2010;25(4):309-17.

4. Sporer KA, Johnson NJ, Yeh CC, Youngblood GM. Can emergency medical dispatch codes predict prehospital interventions for common 9-1-1 call types? Prehosp Emerg Care. 2008;12(4):470-8.

5. Sporer KA, Wilson KG. How well do emergency medical dispatch codes predict prehospital medication administration in a diverse urban community? J Emerg Med. 2013;44(2):413-22. e3

6. Sporer KA, Youngblood GM, Rodriguez RM. The ability of emergency medical dispatch codes of medical complaints to predict ALS prehospital interventions. Prehosp Emerg Care. 2007;11(2):192-8.

7. Shah MN, Bishop P, Lerner EB, Czapranski T, Davis EA. Derivation of emergency medical services dispatch codes associated with low-acuity patients. Prehosp Emerg Care. 2003;7(4):434-9.

8. Shah MN, Bishop P, Lerner EB, Fairbanks RJ, Davis EA. Validation of using EMS dispatch codes to identify low-acuity patients. Prehosp Emerg Care. 2005;9(1):24-31

9. Craig A, Schwartz B, Feldman M. Development of evidence-based dispatch plans to optimize ALS paramedic response in an urban EMS system [abstract]. Prehosp Emerg Care. 2006:10:114.

10. Hinchey P, Myers B, Zalkin J, Lewis R, Garner D. Low acuity EMS dispatch criteria can reliably identify patients without high-acuity illness or injury. Prehosp Emerg Care. 2007;11(1):42-8.

11. Evans R, McGovern R, Birch J, Newbury-Birch D. Which extended paramedic skills are making an impact in emergency care and can be related to the UK paramedic system? A systematic review of the literature. Emerg Med J. 2014;31(7):594-603.

12. Durant $E$, Fahimi J. Factors associated with ambulance use among patients with low-acuity conditions. Prehosp Emerg Care. 2012;16(3):329-37.

13. Dale J, Higgins J, Williams S, Foster T, Snooks H, Crouch R, et al. Computer assisted assessment and advice for "non-serious" 999 ambulance service callers: the potential impact on ambulance despatch. Emerg Med J. 2003; 20(2):178-83.

14. Snooks H, Williams S, Crouch R, Foster T, Hartley-Sharpe C, Dale J. NHS emergency response to 999 calls: alternatives for cases that are neither life threatening nor serious. [review] [29 refs]. BMJ. 2002;325(7359):330-3.

15. Eastwood K, Morgans A, Smith K, Hodgkinson A, Becker G, Stoelwinder J. A novel approach for managing the growing demand for ambulance services by low-acuity patients. Australian Health Review: A Publication Of The Australian Hospital Association. 2015.

16. Dale J, Williams S, Foster T, Higgins J, Snooks H, Crouch R, et al. Safety of telephone consultation for "non-serious" emergency ambulance service patients. Qual Safety Health Care. 2004;13(5):363-73.
17. Studnek J, Thestrup L, Blackwell T, Bagwell B. Utilization of prehospital dispatch protocols to identify low-acuity patients. Prehosp Emerg Care. 2012;16(2):204-9.

18. Smith WR, Culley L, Plorde M, Murray JA, Hearne T, Goldberg P, et al. Emergency medical services telephone referral program: an alternative approach to nonurgent 911 calls. Prehosp Emerg Care. 2001;5(2):174-80.

19. Crowther L, Williams R. Nurse interventions in ambulance command-andcontrol centres. Emerg Nurse. 2009;17:22-5.

20. Turner J, Snooks H, Youren A, Dixon S, Fall D, Gaze S, et al. The costs and benefits of managing some low priority 999 ambulance calls by NHS Direct nurse advisers. Sheffield: The University of Sheffield; 2006.

21. Fox C, Rodriguez C, McSwain NE. EMT telephone triage. EMT J. 1981; 5(6):410-5.

22. Fivaz MC, McQueen J, Barron T, Clawson J, Scott G, Gardett Ml, et al. The distribution of recommended care levels by age, gender, and trauma vs medical classification within the emergency communication nurse system. Ann Emerg Dispatch Response. 2015;3(1):14-20.

23. Eastwood K, Morgans A, Smith K, Stoelwinder J. Secondary triage in prehospital emergency ambulance services: a systematic review. Emerg Med J. 2014;32(6):486-92.

24. Lowthian JA, Cameron PA, Stoelwinder JU, Curtis A, Currell A, Cooke MW, et al. Increasing utilisation of emergency ambulances. Aust Health Rev. 2011; 35(1):63-9. 7p

25. Australian Bureau of Statistics. 3218.0 Regional Population Growth Australia 2012-2013 Canberra: Australian Government; 2015 [updated 2015; cited 20163 February]. Available from: http://www.abs.gov.au/ausstats/abs@.nsf/ Products/3218.0 2012-13 Main+Features Victoria?OpenDocument\#PARALINK3.

26. Australian Bureau of Statistics. Greater Melbourne (GCCSA) Canberra: Australian Government; 2015 [updated 29 June 2015; cited 201622 March]. Available from: http://stat.abs.gov.au/itt/r.jsp?RegionSummary\&region= 2GMEL\&dataset=ABS_REGIONAL_ASGS\&geoconcept=REGION\&measure $=$ MEASURE\&datasetASGS=ABS_REGIONAL_ASGS\&datasetLGA=ABS REGIONAL LGA\&regionLGA=REGION\&regionASGS=REGION

27. Ambulance Victoria. Clinical practice guidelines Doncaster: Ambulance Victoria; 2015 [cited 201629 February ]. Available from: http://www. ambulance.vic.gov.au/Paramedics/Qualified-Paramedic-Training/ClinicalPractice-Guidelines.html.

28. Eastwood K, Smith K, Morgans A, Stoelwinder J. Appropriateness of cases presenting in the emergency department following ambulance service secondary telephone triage: a retrospective cohort study. BMJ Open. 2017 7(10):e016845

29. Ambulance Victoria. Ambulance Victoria Annual Report 2011-2012. Doncaster: Ambulance Victoria; 2012

30. Ambulance Victoria. Delivering our patients the right care, at the right time, at the right place: revised clinical response model evaluation report. Doncaster; 2017

31. Murad H, Fleischman A, Sadetzki S, Geyer O, Freedman LS. Small samples and ordered logistic regression: does it help to collapse categories of outcome? Am Stat. 2003;57(3):155-60.

32. Lund B, Raimi S. Collapsing levels of predictor variables for logistic rgression and weight of evidence coding. MidWest SAS Users Group 2012; 2012 September 16-18; Minneapolis, MN. Minneapolis, MN2012.

33. Tabachnick B, Fidell L. Using multivariate statistics. Boston: Pearson; 2001.

34. IMB Corp. IBM SPSS statistics for windows. 23.0 ed. IBM Corp: Armonk, NY; 2015.

35. Suruda A, Vernon DD, Reading J, Cook L, Nechodom P, Leonard D, et al. Pre-hospital emergency medical services: a population based study of pediatric utilization. Inj Prev. 1999;5(4):294-7.

36. Camasso-Richardson K, Wilde JA, Petrack EM. Medically unnecessary pediatric ambulance transports: a medical taxi service? Acad Emerg Med. 1997:4(12):1137-41.

37. Fessler SJ, Simon HK, Yancey li AH, Colman M, Hirsh DA. How well do general EMS 911 dispatch protocols predict ED resource utilization for pediatric patients? Am J Emerg Med. 2014;32(3):199-202.

38. Spooren D, Buylaert W, Jannes C, Henderick H, Van Heeringen C. Patients with psychiatric emergencies transported by an ambulance in an urban region. European journal of emergency medicine: official journal of the European society for. Emerg Med. 1996;3(1):14.

39. Vine R. Safe transport of people with a mental illness. In: health do, editor. Mental health, drugs and regions division, Victorian Government: Melbourne; 2011. 
40. Branch MH. Protocol for the transport of people with mental illness 2014. In: Health Do, editor. . Melbourne: Department of Health. p. 2014.

41. Cone DC, Schmidt TA, Mann NC, Brown L. Developing research criteria to define medical necessity in emergency medical services. Prehosp Emerg Care. 2004;8(2):116-25.

Submit your next manuscript to BioMed Central and we will help you at every step:

- We accept pre-submission inquiries

- Our selector tool helps you to find the most relevant journal

- We provide round the clock customer support

- Convenient online submission

- Thorough peer review

- Inclusion in PubMed and all major indexing services

- Maximum visibility for your research

Submit your manuscript at www.biomedcentral.com/submit 